\title{
Erratum
}

\section{Seeing Singapore: Portrayal of the city-state in global print media}

\section{Bradley C. Freeman and Trang Nhung Nguyen}

Place Branding and Public Diplomacy (2012) 8, 247. doi:10.1057/pb.2012.16

Correction to: Place Branding and Public Diplomacy (2012) 8(2): 158-169. doi:10.1057/pb.2012.10

The text appearing in the author biography for Trang Nhung Nguyen described her as attending an incorrect university misrepresented as:

Holds B.S. from California State University, Singapore.

The above text should have appeared as:

Holds B.S. from California State Univeristy, Sacramento. 